\title{
Airborne Ultrasonic Vortex Generation Using Flexible Ferroelectrets
}

\author{
Joao L. Ealo, José Carlos Prieto, and Fernando Seco
}

\begin{abstract}
Cellular ferroelectrets exhibit interesting electromechanical-acoustical characteristics. Their recent appearance and remarkable properties open up new possibilities for the design and development of ultrasonic transducers. In particular, the feasibility of fabricating ultrasonic vortex generators using ferroelectret films is demonstrated in this work. To this end, a transducer prototype was built by gluing the material onto a tangential-helical surface (outer diameter: $40 \mathrm{~mm}$, pitch: $3.45 \mathrm{~mm}$ ). Experimental results agree well with the theoretical estimation of the pressure and phase of the acoustic field in the near field and far field, which corroborates the potential of ferroelectrets to customize special acoustic fields. Furthermore, the proposed fabrication procedure is inexpensive and represents a new alternative for exploring and analyzing the special characteristics of acoustical helical wavefronts.
\end{abstract}

\section{INTRODUCTION}

$\mathrm{P}$ HASE distribution singularities constitute an intrinsic characteristic of a given acoustic field. This type of feature occurs naturally in a wavefront and is known to be resistant to disturbances in the field [1]. In general, phase singularities, depending on the morphology of the dislocation, can be axial, screw-type, or mixed. Particularly, in this work we focused on screw dislocations, in which the transverse plane of the wavefront, normal to the propagation axis, exhibits a phase distribution which linearly increases from 0 to $2 \pi$ over any circular trajectory around the principal axis. Fig. 1 shows a spatial representation of a beam with a screw dislocation, also known as a helical wave.

Because of the great similarity between a helical wavefront and vortices in a fluid, pressure fields with screw dislocation are also known as acoustic vortices (AV). Indeed, under special conditions, the interaction between vortices of this type resembles that of their fluid counterparts [2]. Although the existence of a wavefront with screw dislocation was first identified acoustically, most of the subsequent research and applications has been reported in the optics field [3]. In this sense, it has been demonstrated that a helical wavefront transports angular moment [4], which makes it possible to control the orientation of ab- sorbent particles in an optical tweezer [5]. Applications to trap atoms have also been proposed [6].

In the acoustic context, AV can occur either in the linear or nonlinear regime [7], [8]. Considering that several researchers have found analogies between the structure of screw dislocations and vortices in fluids and quantum mechanics, the AV has been proposed as a tool for the experimental study of their optical, fluidic, and quantum counterparts. Recently the transfer of angular momentum from rotating acoustic waves to matter has been experimentally demonstrated by using several sound sources [9], [10]. Another interesting property of the AV is the storage and transmission of digitized information with the particularity that the phase coding is in space and not in time [11]. Even though the potential of use of AV has been reported, their application in acoustics has been scarce. This can be attributed, in part, to the need for complex instrumentation to generate a helical wavefront. Thus far, different methods for AV generation have been proposed, namely, direct generation from a specially shaped source [7], [12], the optoacoustic technique [13], mode conversion from wave plane to AV [9], or use of a phased array system [11].

Ferroelectret technology exhibits interesting properties which make it an excellent candidate to satisfy the current demand for the development of airborne ultrasonic transducers. In particular, cellular ferroelectrets open up new possibilities at the transducer design stage, to approach the trade-off among bandwidth, directivity, sensitivity, and cost. This is possible because of their high $d_{33}$ piezoelectric coefficient; wide frequency range of operation (from $30 \mathrm{kHz}$ to $2 \mathrm{MHz}$ ); low acoustic impedance $(\approx 0.03$ MRayls), which results in a good adaptation to the air; and their unprecedented mechanical flexibility. Furthermore, ferroelectrets are easy to use and have a low fabrication cost [14]-[16]. In this work, we propose a fast and inexpensive alternative for the creation of acoustic vortices by gluing a ferroelectret film onto a tangential/ helical surface substrate. The high mechanical flexibility of this material allows us to fabricate transducers on any developable surface. (A developable surface is any surface that can be obtained by transforming a plane without tearing, stretching or shrinking, i.e., folding, bending, rolling, cutting, and/or gluing.) This characteristic of the film, along with its broadband piston-like response that remains unaltered regardless the radius of curvature, opens up the possibility to customize special acoustic patterns [16], such as acoustic vortices in air and at ultrasonic frequencies. To the best of our knowledge, this is the first demonstration of the generation of $\mathrm{AV}$ at ultrasonic 

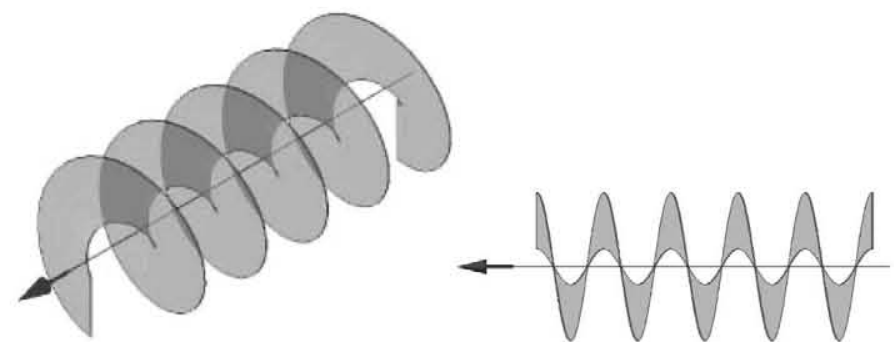

Fig. 1. Representation of the constant phase planes of a wavefront with screw dislocation (helical wavefront). The black arrow indicates the direction of propagation of the wavefront.

frequencies in air. Using broadband ferroelectrets as the active material in the vortex generator fabrication process also constitutes a novelty.

In Section II, a detailed description is given of the AV generator transducer and the instrumentation utilized. In Section III, an overview of the theoretical formulation of helical beams is included for completeness. In Section $\mathrm{IV}$, the generated radiating pattern is measured and compared with simulation results. The results obtained allow us to corroborate the great potential of ferroelectrets to fabricate developable-surface transducers. Finally, the conclusions of the work are presented.

\section{Materials And Methods}

To fabricate our vortex generator prototype, we have used the electromechanical film Emfit, type HS-0320BRAL1 (Emfit Ltd., Vaajakoski, Finland). The film consists of a cellular structure of polypropylene whose internal cavities have been permanently polarized. The nominal thickness of the film is $\approx 70 \mu \mathrm{m}$ and one of its sides is coated with vaporized aluminum, which acts as the upper electrode.

To fabricate the transducer, the Emfit film is cut to shape and size and fixed on a rigid substrate. The nonmetalized side of the active film is glued to the substrate surface using an electrically conductive adhesive tape, which does not affect the electromechanical response of the film and provides a sufficiently rigid and homogeneous foundation. Because the substrate is a developable surface, the creation of creases is avoided. Consequently, the substrate geometry determines the radiating field pattern. The solid substrate geometry was fabricated using a rapid prototyping machine using acrylonitrile butadiene styrene (ABS) polymer.

\section{A. Vortex Generator}

The prototype substrate exhibits a tangential surface which corresponds to a screw dislocation of a pitch $p=$ $3.45 \mathrm{~mm}$, see Fig. 2. In this work, we have measured the acoustic field generated by a burst excitation signal of ten cycles at $100 \mathrm{kHz}$. That is, the resultant vortex exhibits a topological charge $m=p / \lambda=-1$ where $\lambda$ is the acous-
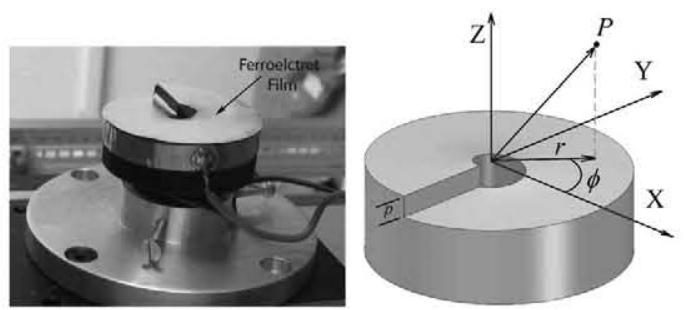

Fig. 2. Left: Prototype fabricated with Emfit film glued on a helical substrate using an electrically conductive adhesive tape. Dislocation $p$ of $1 \lambda$ at $100 \mathrm{kHz}$ in air. Outer diameter: $40 \mathrm{~mm}$. Right: CAD model of the screw dislocation.

tic wavelength. At frequencies greater than $150 \mathrm{kHz}$, the velocity profile of the film becomes uneven because of the random nonuniformities of the electromechanical properties of the film. However, at frequencies up to $100 \mathrm{kHz}$, the vibratory pattern of the Emfit film resembles a piston-like response. Consequently, a quasi-ideal helical wavefront was expected at this frequency [17].

\section{B. Instrumentation Setup}

The acoustic field generated was measured at two different observation planes, perpendicular to the axis of propagation, using a calibrated microphone (1/8 in. Type 4138, Brüel and Kjær, Nærum, Denmark). Specifically, the helical beam was measured at a distance of $100 \mathrm{~mm}$ and $200 \mathrm{~mm}$ from the transducer. Using an XY linear motion stage system, it was possible to measure the instantaneous pressure amplitude at different observation points arranged in a regular square grid. A displacement increment of $1.6 \mathrm{~mm}$ was chosen in both horizontal and vertical directions. To avoid the creation of a standing wave field, a low pulse repetition frequency was used. Also, the transducers were located at a sufficient height from the surface of the antivibratory table to avoid the effect of reflections.

Excitation signals were produced using a signal generator (33120A, Agilent Technologies, Santa Clara, CA) along with a power amplifier (9400, Tabor Electronics, Tel Hanan, Israel), which allows us to apply ac voltages of up to $400 \mathrm{Vpp}$ at frequencies from 0 to $500 \mathrm{kHz}$, see Fig. 3 .

\section{Acoustic Vortex: Theory}

Gauss-Laguerre beams are solutions of the paraxial wave equation in cylindrical coordinates. This type of beam exhibits many of the special characteristics of a helicoidal wave. Because of this, and considering their rather simple mathematical description, they are commonly used to synthesize acoustical vortices [7], [18].

Considering a cylindrical coordinate system $(r, \phi, z)$ with origin $O$ located at the center of the transducer, $z$ indicates the propagation direction normal to the transducer, and $(r, \phi)$ are the cylindrical coordinates of any transversal plane (see Fig. 2). The acoustic pressure at a given point $(r, \phi)$ is given as [19] 


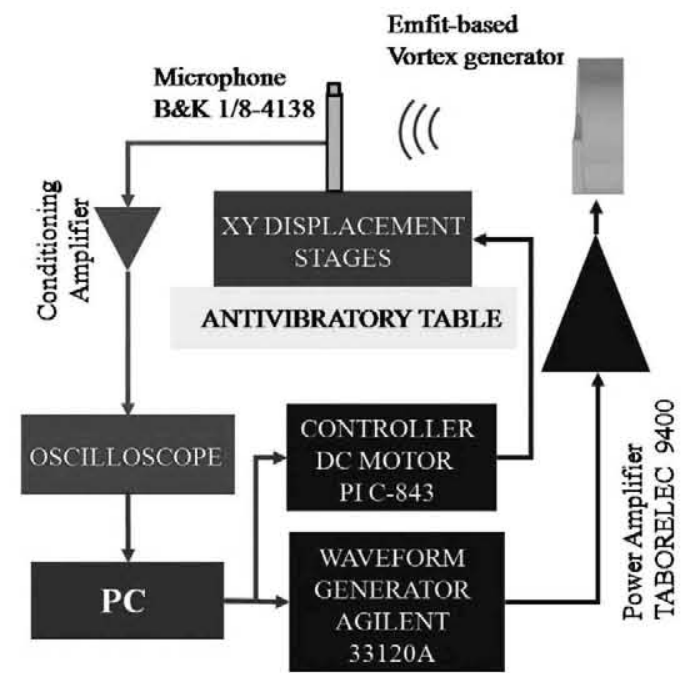

Fig. 3. Instrumentation setup.

$$
P(r, \phi, z, t)=p_{n, m}(r, \phi, z) \exp (i(k z-\omega t)),
$$

where

$$
p_{n, m}(r, \phi, z)=E(r, z) G_{n, m}(r, z) \Phi_{m}(\phi) \Psi_{n}(z)
$$

is the pressure distribution at a plane normal to the propagation axis. The functions $E, G, \Phi$, and $\Psi$ are, respectively, the envelope of the beam (usually assumed to be Gaussian), the amplitude distribution near the core of the vortex, the beam phase structure, and the Gouy's phase.

A Gaussian envelope of the beam is given by

$$
E(r, z)=\frac{A}{\left(1+z^{2} / z_{\mathrm{R}}^{2}\right)^{1 / 2}} \exp \left(\frac{-r^{2}}{w^{2}(z)}\right) \exp \left(-i \frac{k r^{2} z}{2\left(z^{2}+z_{\mathrm{R}}^{2}\right)}\right),
$$

where $A$ is a normalization constant, $z_{\mathrm{R}}=k w_{0}^{2} / 2$ is the Rayleigh's distance ( $w_{0}$ is the width of the beam at $z=0$ and $k$ is the wavenumber), and $w(z)=w_{0}\left[1+\left(z / z_{\mathrm{R}}\right)^{2}\right]^{1 / 2}$ represents the width distribution of the beam throughout the propagation path.

Pressure distribution close to the core of the beam is given by

$$
G_{n, m}(r, z)=\left(\frac{r \sqrt{2}}{w(z)}\right)^{|m|} L_{(n-|m|) / 2}^{|m|}\left(\frac{2 r^{2}}{w^{2}(z)}\right),
$$

where $L_{(n-|m|) / 2}^{|m|}$ are the Laguerre's generalized polynomials [20] of radial index $n=|m|,|m|+2,|m|+4, \ldots$, and topological charge $m$.

The helical structure of the beam phase results from the term:

$$
\Phi_{m}(\phi)=\exp (i m \phi)
$$

and the Gouy's phase reads [7], [21]:

$$
\Psi_{n}(z)=\exp [-i(n+1) \psi(z)]
$$

where $\psi(z)=\arctan \left(z / z_{\mathrm{R}}\right)$.
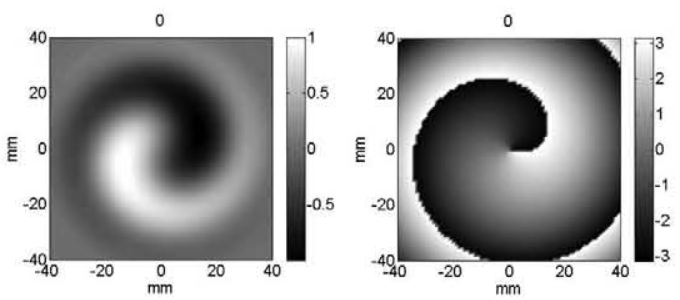

Fig. 4. Instantaneous Gaussian helical field. Simulation results using a topological charge $m=-1$. Left: pressure magnitude, right: phase.
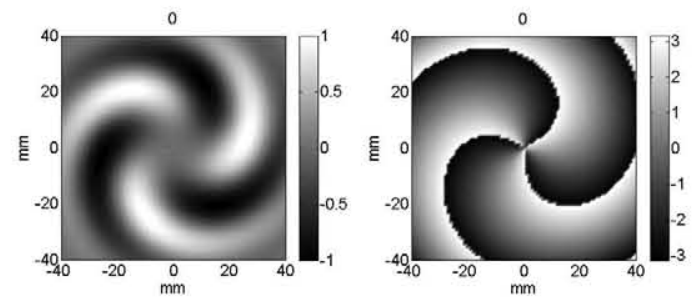

Fig. 5. Instantaneous Gaussian helical field. Simulation results using a topological charge $m=-3$. Left: pressure magnitude, right: phase.

Eq. (1) enables us to synthesize a Gaussian beam corresponding to a phase singularity in the wavefront and its evolution with time at any observation plane parallel to the vortex generator transducer. This result, used frequently to model an optical helical beam, is employed in the next section to qualitatively compare the structure of the screw dislocation obtained from theoretical simulation with that measured at the observation planes. Figs. 4 and 5 show the instantaneous pressure field of a Gaussian beam and its respective phase distribution, for two different topological charges, i.e., $m=-1$ and $m=-3$. Note that phase varies between $-\pi \mathrm{rad}$ and $\pi \mathrm{rad}$ as we follow a circular trajectory around the core of the vortex; finding one and three discontinuities respectively.

Considering that the Gaussian beam formulation is not satisfactory to model the near field pattern of a finite source generating a helical beam, we have also used the well-known distributed point source method [22] to numerically predict the acoustic field produced by the fabricated prototype, as shown in Fig. 6 .

\section{Results: Helical Wavefront Generation}

In this section, we demonstrate that gluing a ferroelectret film on a developable surface allows us to generate a customizable acoustic radiating pattern. In particular, we show that it is possible to create an acoustic vortex using a tangential surface substrate. Empirical and theoretical simulation results are compared to evaluate the quality of the generated helical wavefront at two different planes of observation. We applied a sinusoidal burst of 10 cycles at a frequency of $100 \mathrm{kHz}$, using a low pulse repetition frequency to avoid the creation of a standing wave. Figs. 7 and 8 show the measured and estimated acoustic pressure fields, at 100 and $200 \mathrm{~mm}$, respectively, at 4 different 

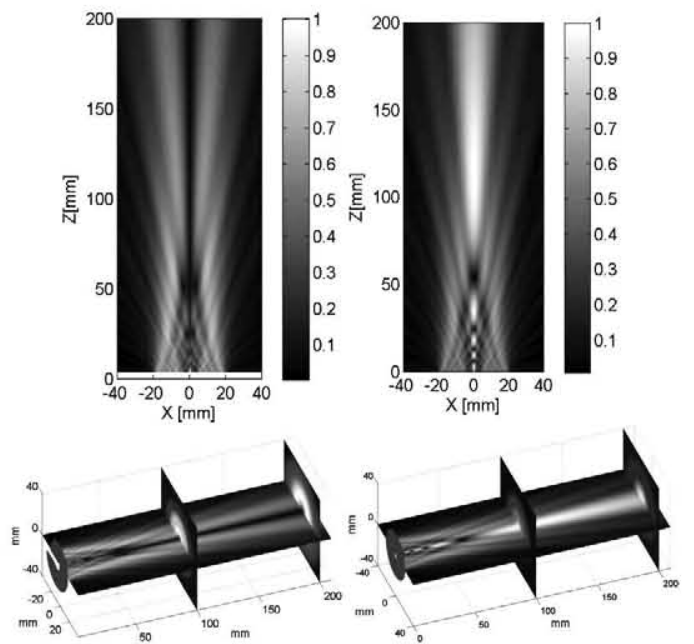

Fig. 6. Magnitude estimation of the azimuthal field (XZ) radiated by a finite source of similar dimensions to the fabricated vortex generator, with (left) and without (right) screw dislocation. Estimation accomplished using the distributed point source method. $m=-1$, frequency $=100 \mathrm{kHz}$.

instants during a cycle of oscillation. Experimental results agree well with the theoretical estimation obtained using a synthesized Gaussian beam of topological charge $m=$ -1 (see also Fig. 4), which corroborates the feasibility of using cellular ferroelectrets to create acoustic vortices.

In Fig. 9, the envelope of the synthesized Gaussian beam is compared with that of the acoustic vortex measured at the observation planes. A fairly good agreement is observed in the far field of the vortex generator prototype, at (X,Y,200 mm). However, in the near field ( $Z$ $\leq 116.61 \mathrm{~mm}$ ), the measured envelope does not properly follow Gaussian behavior. This result is explained by the limitations of the Gaussian beam formulation to emulate the radiated field of a finite acoustic vortex generator (see Fig. 6).

Figs. 10 and 11 depict the stationary absolute value of the pressure distribution along with the instantaneous phase, at 100 and $200 \mathrm{~mm}$ from the transducer, respectively. These estimation results were obtained using the distributed point source method because the observation plane at $Z=100 \mathrm{~mm}$ is still in the near field of the fabricated transducer. The screw dislocation singularity is clearly appreciated because the phase changes from $-\pi$ rad to $\pi$ rad as we move around the core describing a circle. That is, the phase linearly increases from $-\pi$ to $\pi$ as the principal axis is rotated counterclockwise. As expected, the acoustic pressure fields, measured and modeled, resemble a doughnut and the pressure at the core is zero with phase undetermined, even within the near field. It should be pointed out that the effect of the attenuation with frequency was not included in the theoretical simulations.

Finally, the quality of the generated vortex is also observed from Fig. 12. It shows the time variation of the pressure at 51 equidistant observation points deployed on a straight line that crosses the principal axis $(\mathrm{Z})$ and is located in the plane $(\mathrm{X}, \mathrm{Y}, 200 \mathrm{~mm})$. The low pressure level
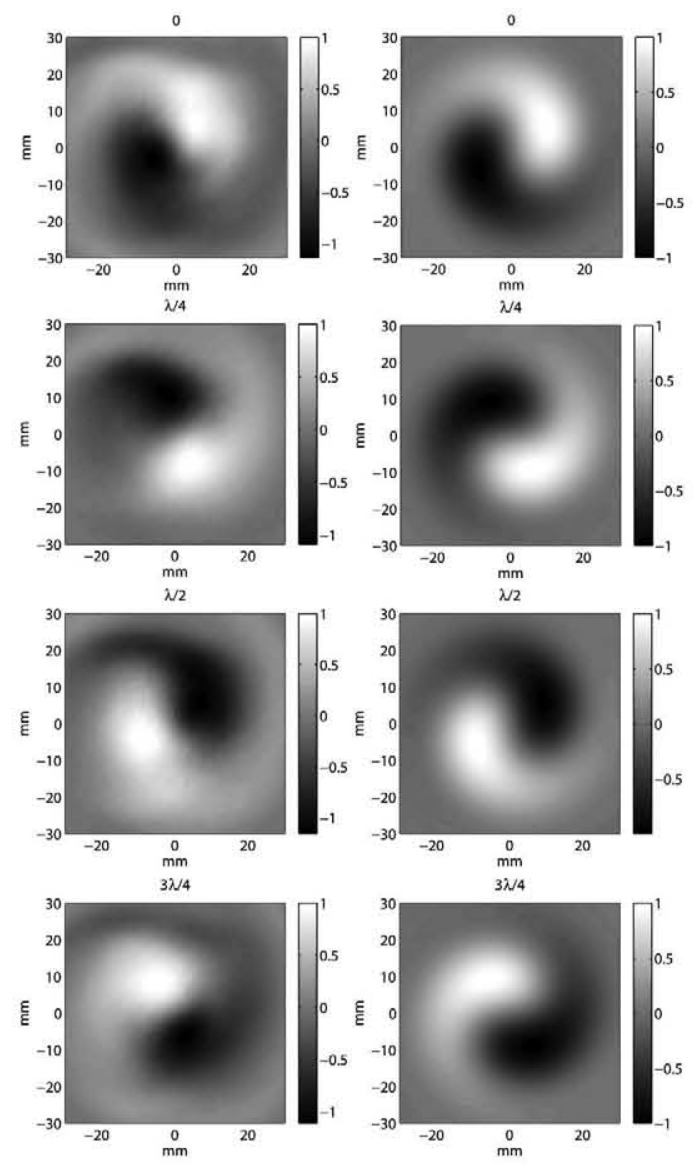

Fig. 7. Normalized instantaneous pressure amplitude at an observation plane located $100 \mathrm{~mm}$ from the transducer. Four different instants within a period of oscillation $\tau$ are shown $(0, \tau / 4, \tau / 2,3 \tau / 4)$. Topological charge $m=-1$. Left: Measured acoustic vortex, right: synthesized Gaussian beam $\left(w_{0}=13 \mathrm{~mm}, z=100 \mathrm{~mm}\right)$.

close to the core is clearly observed; see the time response at point number 25. Also, a phase shift of $\pi$ between any two observation points located on opposite sides of the center is measured. Fig. 13 illustrates the points of constant phase located on the observation plane (X, Y, $200 \mathrm{~mm}$ ), at every sample time, i.e., as the measured wavefront crosses the plane. The helical deployment of the points observed also allows us to conclude that the generated field greatly emulates the theoretical characteristics of an ideal acoustic vortex, see also Fig. 1.

\section{CONCLUSIONS}

Cellular ferroelectrets exhibit special characteristics which result in an unprecedented versatility at the design stage of acoustic transducers. A high mechanical flexibility, which allows us to fabricate transducers on any developable surface, along with a broadband piston-like response that remains unaltered regardless of the radii of curvature, open up the possibility of creating special acoustic patterns such as acoustic vortices in air and at ultrasonic frequencies. To our knowledge, this has not yet been reported. 

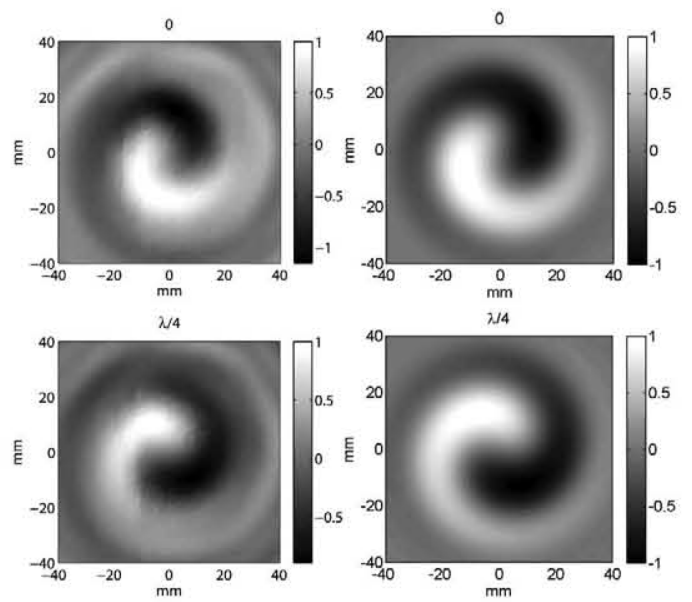

$N / 2$

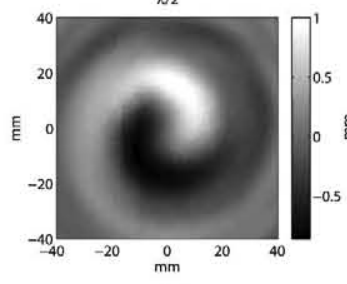

$3 N 4$
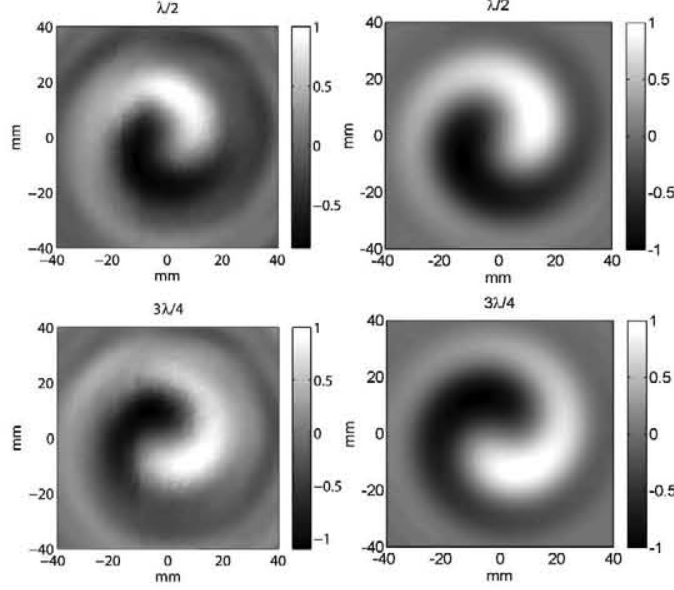

$3 \pi / 4$

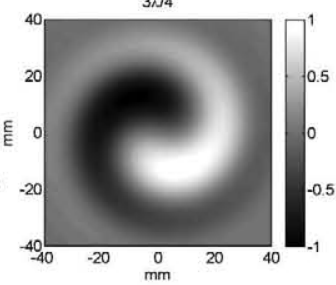

Fig. 8. Normalized instantaneous pressure amplitude at an observation plane located $200 \mathrm{~mm}$ from the transducer. Four different instants within a period of oscillation $\tau$ are shown $(0, \tau / 4, \tau / 2,3 \tau / 4)$. Topological charge $m=-1$, left: measured acoustic vortex (E). Right: synthesized Gaussian beam $\left(w_{0}=13 \mathrm{~mm}, z=200 \mathrm{~mm}\right)$.
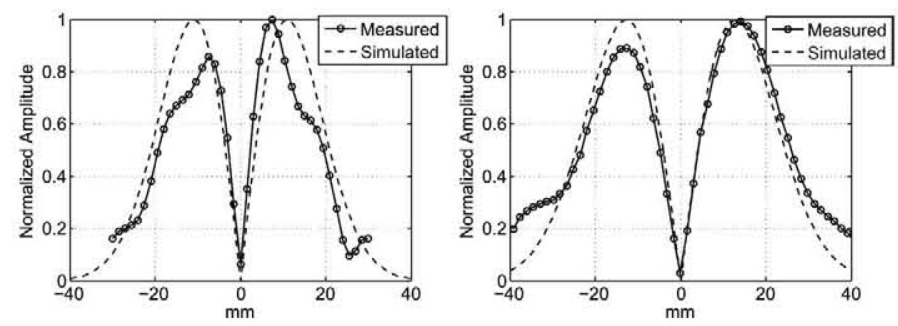

Fig. 9. Comparison between the envelope of a Gaussian beam and that of the acoustic vortex generated with the ferroelectret-based transducer. Left: at the observation plane (X, Y,100 mm), i.e., in the near field of the vortex generator; right: at the observation plane (X,Y, $200 \mathrm{~mm}$ ).

Experimental results obtained were in excellent agreement with theoretical estimations which indicates that it is possible to generate high-quality helical wavefronts easily and reliably. This paves the way for the potential use of acoustic vortices in engineering applications without using a complicated setup to produce them. Considering that the sensitivity of the ferroelectret films is moderate (around 15 to $20 \mathrm{~dB}$ lower than that of resonant piezoceramics and PVDF-based transducers), the excitation voltage and
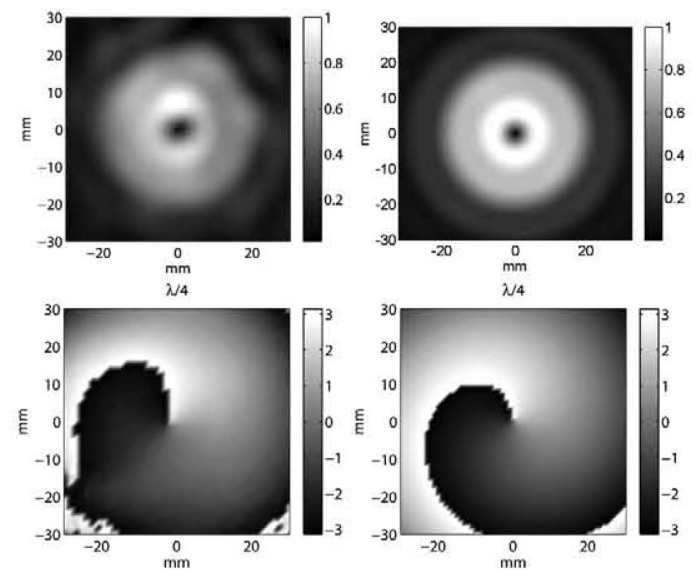

Fig. 10. Normalized magnitude (top panel) and phase (low pane) of a helical wavefront at an observation plane located at a distance of $100 \mathrm{~mm}$ from the transducer. Maximum pressure obtained: 1.37 Pa. Frequency $=100 \mathrm{kHz}$. Topological charge $m=-1$. Left: measured acoustic vortex, right: simulated using the distributed point source model method.
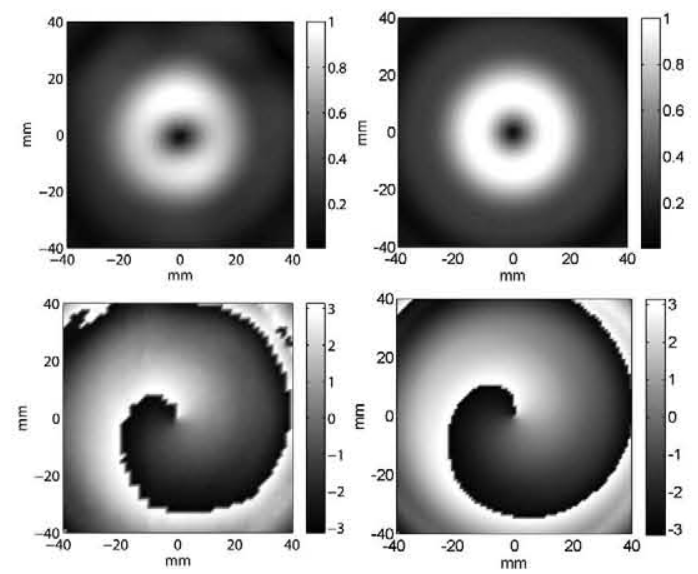

Fig. 11. Normalized magnitude (top panel) and phase (low pane) of a helical wavefront at an observation plane located at a distance of $200 \mathrm{~mm}$ from the transducer. Maximum pressure obtained: $0.98 \mathrm{~Pa}$, frequency $=100 \mathrm{kHz}$. Topological charge $m=-1$. Left: Measured acoustic vortex (E), right: simulated using the distributed point source model method.

the size of the vortex generator must be properly chosen to obtain the desired acoustic output. However, its wide frequency range of operation, from $20 \mathrm{kHz}$ to several hundreds of kilohertz, which allows us to create vortices of different topological charge using the same transducer, and its ease of use make the ferroelectret technology an excellent candidate for ultrasonic vortex generation. Further research is required to quantify the effect of the variations in the piston-like response of the film at frequencies beyond $150 \mathrm{kHz}$ on the generated acoustic vortices.

It is important to point out that the ultrasonic vortex can also be achieved using a ferroelectret-based multitransducer along a phased-array system. In this manner, the phase singularity is created not by using a helical surface but electronically. An easy procedure to fabricate ferroelectret-based multi-transducers has recently been demonstrated [23]. 

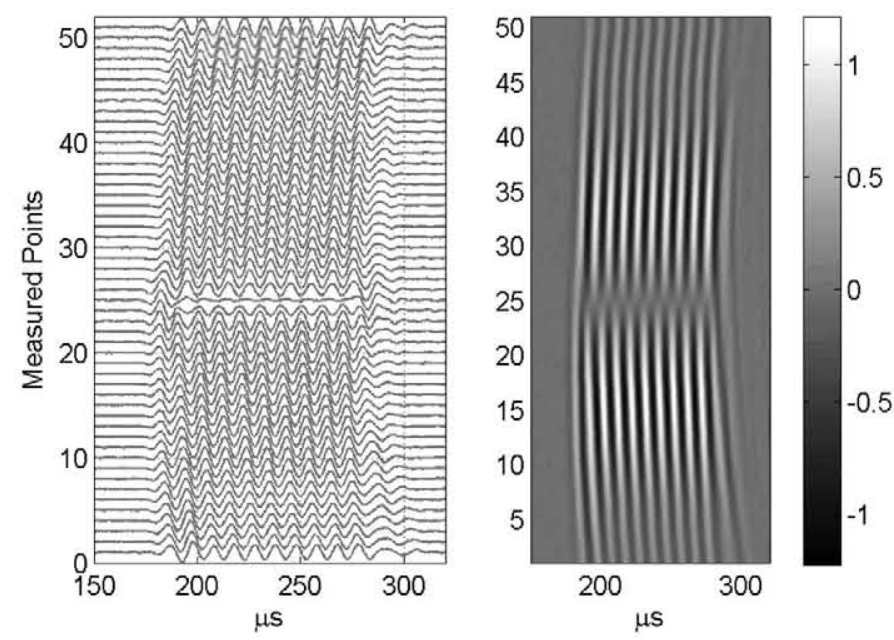

Fig. 12. Time variation of the acoustic pressure of 51 different points deployed on a line that transversely crosses the helical wavefront measured at $(\mathrm{X}, \mathrm{Y}, 200 \mathrm{~mm})$. The spacing of the observation points was $1.6 \mathrm{~mm}$. Left: time responses arranged vertically, right: top view of the pressure distribution.

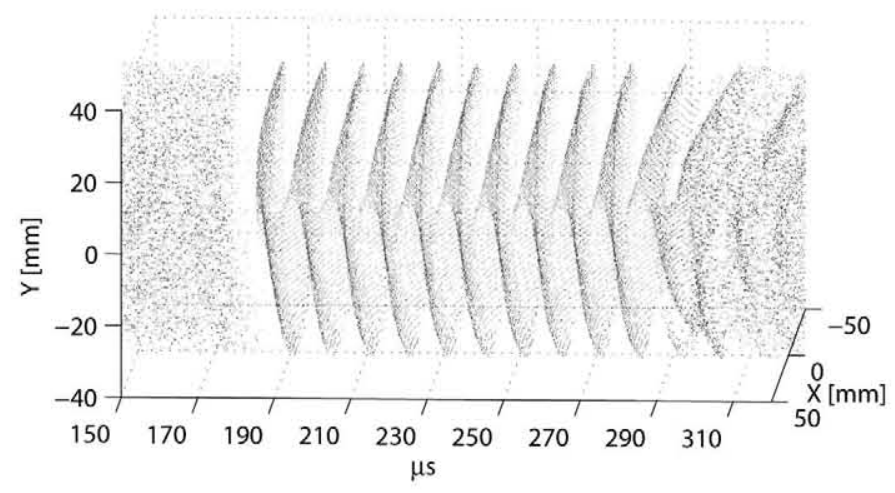

Fig. 13. Time evolution of the ultrasonic vortex. Plotted dots correspond to the points with constant phase measured on a grid covering the plane $(\mathrm{X}, \mathrm{Y}, 200 \mathrm{~mm})$. The spacing between the elements of the grid is $1.6 \mathrm{~mm}$.

\section{REFERENCES}

[1] J. F. Nye, Natural Focusing and Fine Structure of Light, Bristol, UK: IOP Publishing, 1999.

[2] D. Rozas, C. T. Law, and G. A. Swartzlander, "Experimental observation of fluidlike motion of optical vortices," Phys. Rev. Lett., vol. 79, no. 18, pp. 3054-3065, 1997.

3] V. Y. Bazhenov, M. S. Soskin, and M. V. Vasnetsov, "Screw dislocations in light wavefronts," J. Mod. Opt., vol. 39, no. 5, pp. 985-990, 1992.

[4] L. Allen, M. W. Beijersbergen, R. J. C. Spreeuw, and J. P. Woerdman, "Orbital angular momentum of light and the transformation of Laguerre-Gaussian laser modes," Phys. Rev. A, vol. 45, no. 11, pp. 8185-8189, Jun. 1992.

[5] H. He, M. Firese, N. Heckenberg, and H. Rubinsztein-Dunlop, "Optical particle trapping with higher-order doughnut beams produced using high efficiency computer generated holograms," J. Mod. Opt., vol. 42, no. 1, pp. 217-223, 1995.

6] T. Kuga, Y. Torii, N. Shiokawa, T. Hirano, Y. Shimizu, and H. Sasada, "Novel optical trap of atoms with a doughnut beam," Phys. Rev. Lett., vol. 78, no. 25, pp. 4713-4716, 1999.

[7] B. T. Hefner and P. Marston, "An acoustical helicoidal wave transducer with applications for the alignment of ultrasonic and underwater systems," J. Acoust. Soc. Am., vol. 106, no. 6, pp. 3313-3316, 1999.
[8] J. Thomas and R. Marchiano, "Pseudo angular momentum and topological charge conservation for nonlinear acoustical vortices," Phys. Rev. Lett., vol. 91, no. 24, art. no. 244302, 2003.

[9] K. Volke-Sepúlveda, A. O. Santillan, and R. R. Boullosa, "Transfer of angular momentum to matter from acoustical vortices in free space," Phys. Rev. Lett., vol. 100, no. 2, art. no. 024302, 2008.

[10] A. O. Santillán and K. Volke-Sepúlveda, "A demonstration of rotating sound waves in free space and the transfer of their angular momentum to matter," Am. J. Phys., vol. 77, no. 3, pp. 209 215, 2009.

[11] R. Marchiano and J.-L. Thomas, "Doing arithmetic with nonlinear acoustic vortices," Phys. Rev. Lett., vol. 101, no. 6, art. no. 064301, 2008.

[12] HefnerB.MarstonP., "Acoustical helicoidal waves and LaguerreGaussian beams: Applications to scattering and to angular momentum transport," J. Acoust. Soc. Am., vol. 103, no. 5, p. 2971, 1998.

[13] GspanS.MeyerA.BernetS.Ritsch-MarteM., "Optoacoustic generation of a helicoidal ultrasonic beam," J. Acoust. Soc. Am., vol. 115, no. 3, pp. $1142-1146,2004$.

[14] S. Wilson, R. Jourdain, Q. Zhang, and R. Dorey, "New materials for micro-scale sensors and actuators: An engineering review," Mater. Sci. Eng. R, vol. 56, no. 1-6, pp. 1-129, 2007.

[15] M. Dansachmuller, I. Minev, P. Bartu, I. Graz, N. Arnold, and S. Bauer, "Generation and detection of broadband airborne ultrasound with cellular ferroelectrets," Appl. Phys. Lett., vol. 91, no. 22, art. no. $222906,2007$.

[16] J. Ealo, F. Seco, C. Prieto, A. Jiménez, J. Roa, A. Koutsou, and J. Guevara, "Customizable field airborne ultrasonic transducers based on electromechanical film," in IEEE Ultrasonics Symp., 2008. pp. 879-882.

[17] J. Ealo, F. Seco, and A. R. Jiménez, "Broadband EMFi-based transducers for ultrasonic air applications," IEEE Trans. Ultrason. Ferroelectr. Freq. Control, vol. 55, no. 4, pp. 919 929, 2008.

[18] E. Gálvez, "Gaussian beams in the optics course," Am. J. Phys., vol. 74, no. 4, pp. 355-361, 2006.

[19] R. Piestun, Y. Y. Schechner, and J. Shamir, "Propagation-invariant wave fields with finite energy," J. Opt. Soc. Am. A, vol. 17, no. 2, pp. 294-303, 2000.

[20] M. Abramowitz and I. Stegun, Handbook of Mathematical Functions, 9th ed., Mineola, NY: Dover, 1970.

[21] R. Marchiano and J.-L. Thomas, "Synthesis and analysis of linear and nonlinear acoustical vortices," Phys. Rev. E, vol. 71, no. 6, art. no. $066616,2005$.

[22] D. Placko and T. Kundu, DPSM for Modeling Engineering Problems. New York, NY: Wiley-Interscience, 2007.

[23] J. Ealo, J. Camacho, and C. Fritsch, "Airborne ultrasonic phased arrays using ferroelectrets: A new fabrication approach," IEEE Trans. Ultrason. Ferroelectr. Freq. Control, vol. 56, no. 4, pp. 848-858, 2009. 


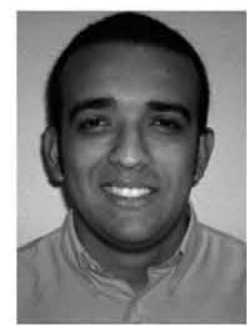

Joao L. Ealo is an Assistant Professor in the School of Mechanical Engineering of the University of Valle, Cali, Colombia. He was born in Cartagena de Indias, Colombia, in 1976. He received a B.Sc. degree in mechanical engineering from the University of Ibagué, Colombia, in 1998, and an M.Sc. degree in industrial control systems from the University of Valladolid, Spain in 2000. He obtained a $\mathrm{Ph} . \mathrm{D}$. degree in mechanical engineering from the Polytechnic University of Madrid, Spain, in 2009.

His current research interests are aimed at exploring the potential of different transducer technologies to be used in air-coupled ultrasonic applications, such as acoustic imaging, nondestructive testing, material characterization, robot navigation, etc. This comprises the design, fabrication, modeling, and electro-mechanical-acoustical characterization of transducers.

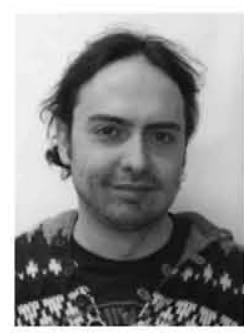

José Carlos Prieto was born in León, Spain, in 1978. He received a Technical degree in industrial electronics and a B.S. degree in electronics engineering from the Universidad de Extremadura, Badajoz, Spain, in 1999 and 2003, respectively, and a Master's degree in robotics from the Universidad Politécnica de Madrid, Madrid, Spain, in 2007. He is currently working toward a Doctoral degree in robotics with the Universidad de Alcalá, Madrid.
Since 2004, he has been a Researcher with the Instituto de Automática Industrial, Consejo Superior de Investigaciones Científicas (CSIC), Madrid. His research interests are focused on localization systems, mainly those based on ultrasonic signals, with special emphasis on signal design and processing, positioning algorithms, robustness, standardization, optimal configurations, calibration methods, and development of new transducers.

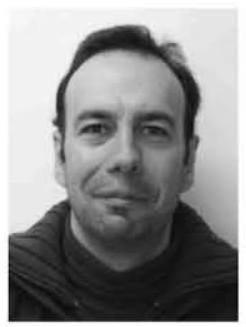

Fernando Seco Granja was born in 1972 in Madrid, Spain. He holds a degree in physics (Universidad Complutense of Madrid, 1996) and a Ph.D. degree in physics (UNED, 2002), for which his dissertation dealt with the magnetostrictive generation of ultrasonic waves applied to a linear position sensor. Since 1997, he has been working at the Consejo Superior de Investigaciones Científicas (CSIC) in Arganda del Rey, Madrid, where he holds a research position.

His main research interest is in the design and development of indoor local positioning systems (LPS), especially those based on ultrasonic and radiofrequency technologies, in the modulation and codification of ultrasonic signals, and in Bayesian localization methods. 